\title{
Spectrum Analysis of Wireless Electromyography in Water and on Dry Land: A Single Case Example
}

\author{
Jonas Martens $^{1, *}$, Luc Janssens ${ }^{2,3}$, Filip Staes ${ }^{3}$, Bart Dingenen $^{3}$ and Daniel Daly ${ }^{1}$ \\ ${ }^{I}$ Faculty of Kinesiology and Rehabilitation Sciences, Department of Kinesiology, KU Leuven, Leuven, Belgium \\ ${ }^{2}$ Group T-International University College Leuven, Leuven, Belgium \\ ${ }^{3}$ Faculty of Kinesiology and Rehabilitation Sciences, Department of Rehabilitation Sciences, KU Leuven, Leuven, \\ Belgium
}

\begin{abstract}
Recent developments in wireless capture of muscle activity signals have improved the freedom of motion and the ease of use. Nevertheless, no study has examined the quality of wireless electromyographic technology during water activities. In this study the performance of a surface electromyography (SEMG) wireless system on dry land and in water was compared. Tibialis anterior muscle activity of 1 subject while contracting against a resistance of 84 Newton $(\mathrm{N})$ was measured in 2 conditions: using a wireless (KINE) system with waterproof taping on the pool deck and submerged. Analysis of the raw signal showed acceptable ratios of 37 to 44 decibel $(\mathrm{dB})$ between rest activity and the force activity at $84 \mathrm{~N}$ in both conditions. The total spectrum analysis and short term frequency analysis showed that no filtering occurred in either of the two conditions as frequencies were picked up in the entire spectrum. Spectrum analysis of rest activity or of the force buildup showed no spikes indicating any pickup of noise. The wireless system produced clean results and submersion and as a consequence recollection from the memory of the unit did not cause additional noise. Furthermore, the wireless system provided a satisfactory signal-to-rest activity ratio of approximately $40 \mathrm{~dB}$ and a range of 4 millivolts $(\mathrm{mV})$. The KINE EMG units provided a valid means to analyze muscle activity during swimming while permitting unrestricted movement and ease of use.
\end{abstract}

Keywords: Aquatics, electromyography, spectrum analysis, submersion, wireless technology.

\section{INTRODUCTION}

Muscle activity during swimming has long been of interest to both coaches and researchers. Between the publication of Curetons Mechanics and kinesiology of swimming [1] in 1930 and 1964, attempts have been made to describe the muscles anatomical function and involvement in swimming. Muscle involvement is however only one element. The muscle use pattern within a complex rhythmical swimming movement is another important component. This information cannot be obtained by functional anatomical deduction [2]. The first study of myoelectric signals during swimming was published in 1964 [3]. In this study, the activity of 15 muscles in all 4 competitive swimming strokes was examined and compared between 9 Olympic and 5 university team swimmers. It was concluded that the muscle activation pattern of top swimmers was more effective and that to move the body forward in crawl, breaststroke and butterfly, the extensors of arm and trunk contracted more vigorously than the flexors.

*Address correspondence to this author at the Faculty of Kinesiology and Rehabilitation Sciences, Department of Kinesiology, KU Leuven, Tervuursevest 101 bus 1500, 3001, Leuven, Belgium;

Tel: 0032163291 04; E-mail: Jonas.Martens@ faber.kuleuven.be
Backstroke was an exception. In this stroke, the opposite pattern was found.

The vast majority of studies published used amplitude analysis methods e.g. integrating the raw signal or calculating the root mean square to describe muscle activation patterns in the 4 competitive swimming strokes and/or to compare the muscle activation pattern of a certain group of swimmers to another group. Recently an extensive review of this work has been presented by Clarys and Rouard [4]. In 2006, the first study using frequency analysis to describe muscle fatigue was published [5].

From the beginning, the study of muscle activity during movement in water has been a technical challenge. In the past, three distinct methods of recording muscle potentials in water were used: 1) surface electrodes and wire transmission [3], 2) surface electrodes and telemetric transmission [6] and 3) fine-wire electrodes [7]. Most electromyographic (EMG) studies in swimming have been carried out with surface electrodes, accepting the possible crosstalk of neighboring muscles as part of the data acquisition [4] and other confounding factors related to water immersion such as water interfering with the electrical signal during measurements and leaks between the skin and the surface electrode [8-10]. These findings lead to the generally 


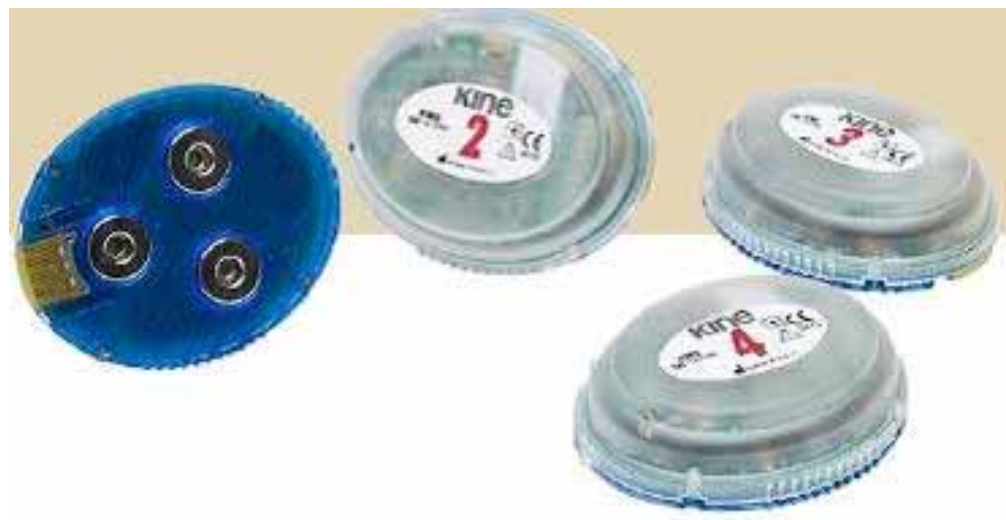

Fig. (1). Wireless electrode units $(5.4 \mathrm{~cm}$ by $4.5 \mathrm{~cm})$.

accepted idea that surface electrodes should be waterproofed to obtain a clear EMG signal. Consequently most studies used some kind of waterproof technique although not always described in detail. Fine wire equipment does not have this problem of leaking or water interfering with the electrode since the electrode is applied subcutaneous, nonetheless the drawbacks of using fine-wired electrodes in swimming studies are the difficult search for subjects willing to swim with this invasive equipment and the fact that leads easily become inoperable [11]. The fine wire technique is mainly used to examine "deep muscles" such as the rotator cuff.

After experiencing numerous difficulties with wired surface EMG (SEMG) systems such as movement restriction during swimming, the introduction of telemetric methods in the 1970's brought about a significant evolution. The swimmer could now move more freely. The most recent developments in wireless muscle activity capture technology again improved the swimmer's freedom of motion and the ease of use for the researcher. To the authors knowledge however, no study has yet been published that addresses the specific issues of the use of wireless technology in the field of electromyography during water activities. The aim of this report is to compare the performance of a surface EMG wireless system on dry land and in water and to examine the specific characteristics of the signal produced by the wireless system available. A positive evaluation will establish the validity of further research on the swimming movement with this equipment.

\section{MATERIALS AND METHODS}

\subsection{Participant}

One 30 year old male subject with a fat percentage of $12,08 \%$, calculated from skinfold measurements, agreed to participate in this study. The participant signed an informed consent that was approved by the Commissie voor Medische Ethiek of the University Hospital of Leuven, which acts as the central commission for this project. The participant did not take any medication.

\subsection{Surface EMG Recording}

To determine if a wireless SEMG system provides altered signal output on dry land and in water, one channel of a 12-channel electromyograph $\left(\mathrm{KINE}^{\circledR}, \mathrm{KINE}\right.$ Ltd., Hafnarfjördur, Iceland) was used. The input impedance was $10 \mathrm{G} \Omega$. This high input impedance made the system less affected by skin resistance. The common mode rejection ratio of $110 \mathrm{~dB}$ guaranteed the suppression of common mode signals on the body and an A/D converter of 10 bit with a range of $4 \mathrm{mV}$ was used. Consequently the sensitivity of this system was $4 \mu \mathrm{V}$. The electrodes (including the reference electrode) of this wireless system were built into a single unit $(26 \mathrm{~g})$ in a triode configuration as can be seen in Fig. (1), with a center-to-center inter-electrode distance of $20 \mathrm{~mm}$. This triode configuration of two signal and one reference wire for each electrode ensured satisfactory common mode voltage suppression between electrodes. The output voltage of an electrode setup is the result of the difference in voltage between each signal electrode and the reference electrode plus a common mode signal which is also present between the electrodes. This is considered as noise on the signal. By positioning the reference electrode adjacent and with equal distance to the signal electrodes, this common mode noise signal is minimized and the output EMG voltage, present between the signal electrodes, will contain less noise. EMG signals were streamed live to a computer with a transmission carrier frequency of $433.05-434.79 \mathrm{MHz}$. When the distance between electrode and receiver was larger than 50 meters or when the electrode was submerged, live signal was lost. In these cases, the KINE system automatically stored the EMG signal in an internal memory of maximal 7 minutes built into the electrode itself. EMG data was collected live, or recollected from the internal memory at a sample rate of $1600 \mathrm{~Hz}$ with KINE Pro software (KINE Ltd., Hafnarfjördur, Iceland).

\subsection{Experimental Procedure}

To compare the system on dry land and in water mediums, muscle activity of the left tibialis anterior was measured in 2 conditions:

- Supine position on a table on the pool deck using the wireless KINE system with waterproof taping,

- Supine position on a table under water so that only the participant's face remained dry using the wireless KINE system with waterproof taping. 


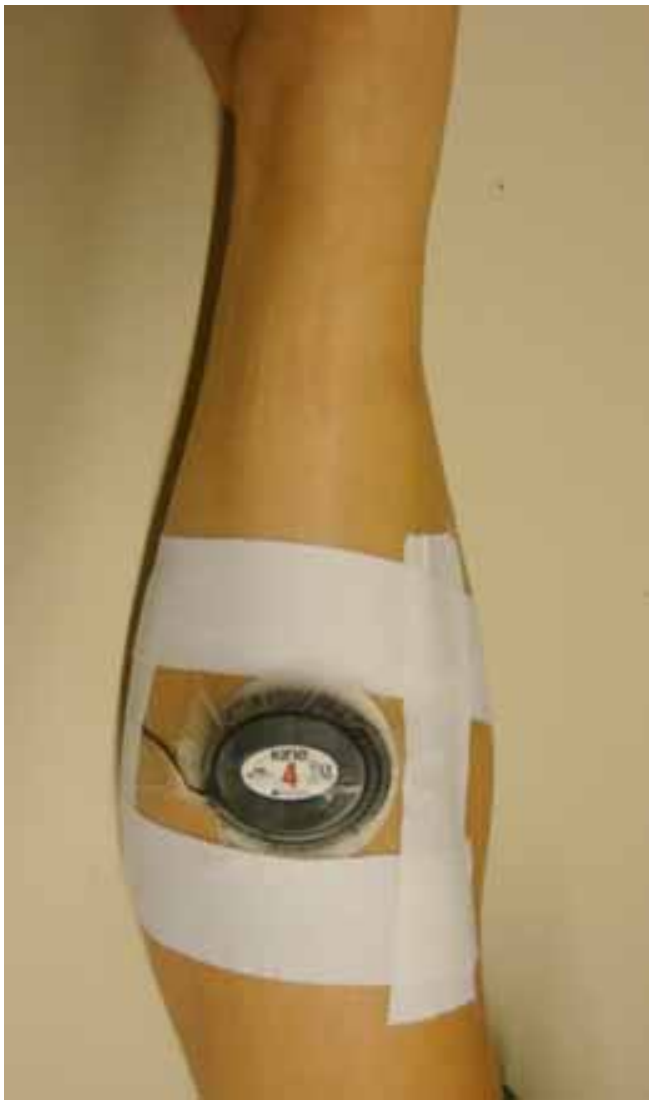

Fig. (2). Waterproof taping of the left tibialis anterior with tip of the antenna sticking out of the taping.

The distance between the electrodes and receiver was never larger than 10 meters, but live wireless transmission was lost in the underwater condition so in that case the EMG signal was stored in the internal memory of the EMG unit and recollected after the trial. The participant performed 3 trials of 15 seconds in the 2 conditions. During the first 5 seconds of each trial the participant relaxed to define the rest EMG. The next five seconds the participant performed a dorsal flexion of the foot against a handheld dynamometer (Hoggan Health Industries, Salt Lake City, Utah, USA) building up the force output to 84 Newton $(\mathrm{N})$. The final 5 seconds the participant held the force output at $84 \mathrm{~N}$ so a clear signal to rest potential could be measured. The trial that showed the most stable rise in Root Mean Square (RMS) was selected for further analysis.

There are a number of calculations that give insight into the amplitude of an EMG signal. Despite the fact that there are a number of complex anatomical, physiological, detection and computational reasons and factors as to why the amplitude of an EMG signal does not directly correlate with the force generated, RMS is considered the most meaningful, since it gives a measure of the signal power [12]. Based on the results of a maximal voluntary contraction $(\mathrm{MVC}=168 \mathrm{~N}), 84 \mathrm{~N}$ represented $50 \%$ of maximal for this participant MVC and was therefore easily repeatable in the two experimental settings. A similar dynamometer was used by Pöyhönen et al. [14] to measure submaximal force output in a study comparing muscle activity in water and on dry land.
Maximal voluntary contractions are subjective and would not have allowed comparison between equipment as the total force output in Newton would have varied. Furthermore, Masumoto and Mercer [13] stated that there seemed to be differences in EMG during maximal voluntary contractions in water and on dry land. In addition, the aim of this study was not to make an analysis of EMG amplitude or of interindividual differences.

Waterproof taping of the KINE EMG unit was achieved by carefully covering the unit with Opsite Flexifix clear film (Smith \& Nephew ${ }^{\circledR}$, London, UK) and then covering the edges with sports tape as shown in Fig. (2). To keep skin impedance low, the site for electrode placement was prepared by shaving, gently abrading the skin using sandpaper, and cleaning with $70 \%$ isopropyl alcohol. Electrodes were positioned and waterproofed in all conditions by the same researcher following the SENIAM guidelines (Surface Electromyography for the Non-Invasive Assessment of Muscles) [15].

\subsection{Data Processing}

After removing the possible zero offset caused by the amplifiers, raw data were processed with Labview Software (National Instruments ${ }^{\circledR}$, Austin, TX, USA). Root mean square was obtained for a running window of $100 \mathrm{~ms}$. Spectrum analysis was performed on the total spectrum, a 2 seconds window during rest activity of the muscle and a 2 seconds window during the buildup of force. A 2 second window was chosen as in this timespan, enough information can be obtained to execute a frequency analysis. The ratio of maximal activity versus the minimal activity corresponding with a force output of $84 \mathrm{~N}$ was automatically obtained by a self-written Labview protocol during a 1 second window of respectively the rest activity, and the maximal activity found during the trial. Here, a 1 second window was chosen because a stable signal is needed also during the maximal activity of the trail. This ratio was expressed as maximal versus minimal activity and in $\mathrm{dB}$.

\section{RESULTS}

Analysis of the raw signal (Table 1, row 1) showed good ratios of 37 to $44 \mathrm{~dB}$ between rest activity and the force activity at $84 \mathrm{~N}$ in both conditions. The total spectrum analysis (Table 1, row 2) showed that no filtering occurred in either of the two conditions as frequencies were picked up in the entire spectrum. The short term frequency analysis (table 1 row 6) confirmed this finding as filtering would have resulted in horizontal bars at certain frequencies. Spectrum analysis of rest activity (Table $\mathbf{1}$, row 3), or of the force buildup (Table 1, rows 4 and 5) showed no spikes indicating any pickup of noise.

\section{DISCUSSION}

The wireless KINE system did not pick up or generate noise and had no built in filters. No differences could be detected in the spectrum analysis between both KINE conditions, so there was no influence on the signal when the live signal was lost once the electrode was submerged. No 
Table 1. Analysis of a $15 \mathrm{sec}$ relaxation and muscle contraction cycle on dry land and in water.

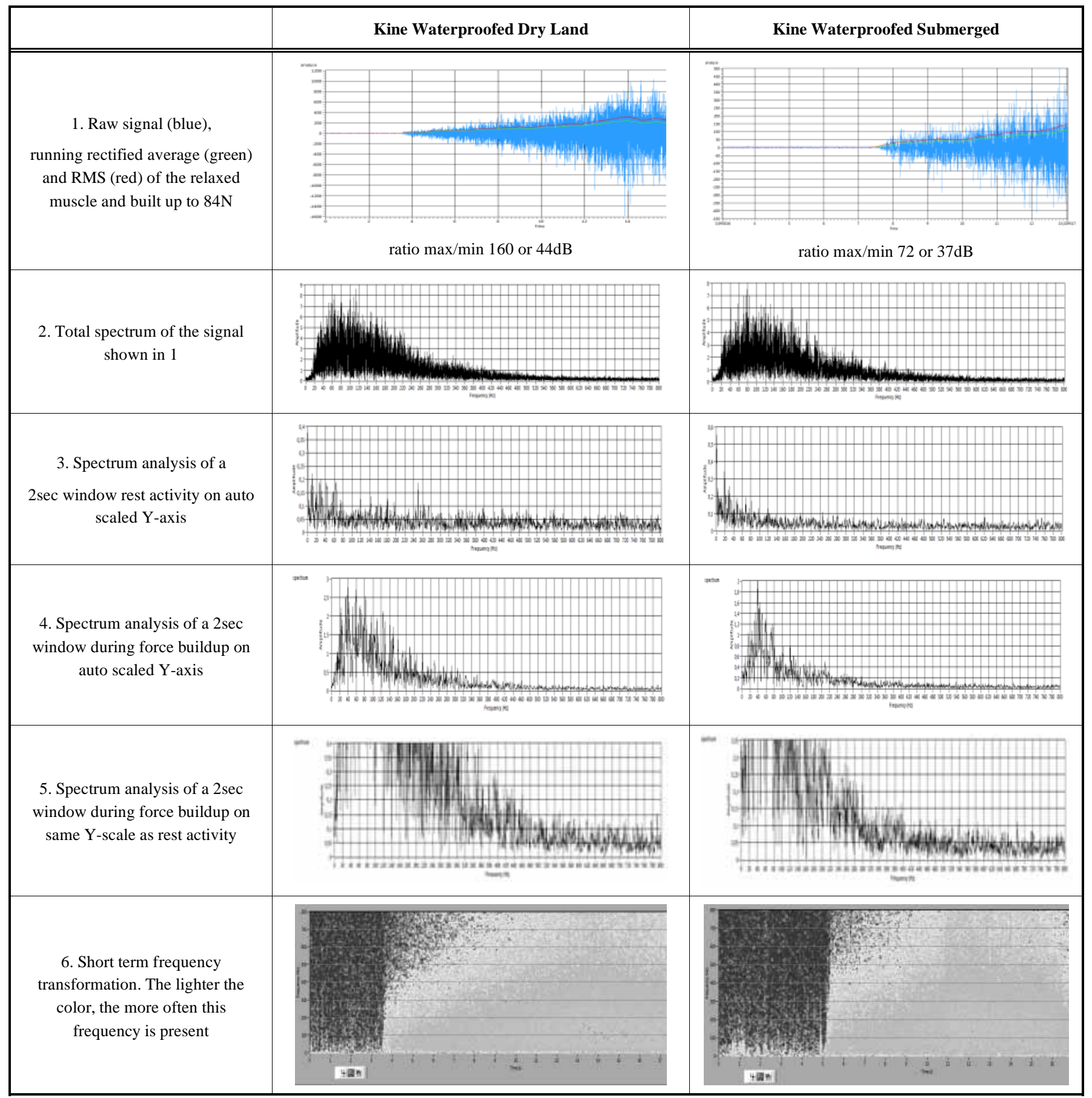

data was lost when recollecting from the memory of the unit. The KINE system provided a clear and noise free signal in the range of $5 \mathrm{~Hz}$ to $500 \mathrm{~Hz}$ where all muscle activity occurs during regular occupational or sport activities in general [16] and future swimming studies in particular are situated. Since the sensitivity of the KINE system is $4 \mu \mathrm{V}$ and a 10 bit converter is used, the range is $4 \mathrm{mV}$. This is sufficient for measuring the total muscle activity during dynamic movements such as swimming. During swimming, voltages rarely exceed $3 \mathrm{mV}[4,17,18]$.

\section{CONCLUSION}

The aim of this study was to compare a SEMG wireless system on dry land and in water. Since wireless EMG technology has only recently become commercially available, this study was the first of its kind. Results showed that the wireless system produced clean results and that submersion and as a consequence recollection from the memory of the unit did not cause additional noise. Furthermore, the wireless system provided a satisfactory signal-to-rest activity ratio of approximately $40 \mathrm{~dB}$ and a 
range of $4 \mathrm{mV}$. The KINE EMG units provided a valid means to analyze muscle activity in water and could be tested in future projects during swimming which would permit unrestricted movement for the swimmer and ease of use for the researcher.

\section{CONFLICT OF INTEREST}

This project was funded by KU Leuven, Faculty of Kinesiology and Rehabilitation Sciences. There is no conflict of interest.

\section{ACKNOWLEDGEMENTS}

This project was financed by KU Leuven, Faculty of Kinesiology and Rehabilitation Sciences. The authors are thankful to both the department of Kinesiology and the department of Rehabilitation Sciences, as well as Group T International University College Leuven.

\section{REFERENCES}

[1] Cureton TK. Mechanics and kinesiology of swimming. Res Quart Am Phys Educ Assoc 1930; 1(4): 87-121.

[2] Clarys JP. A review of EMG in swimming: explanation of facts and/or feedback information. In: Hollander AP, Huijing PA, de Groot G, Eds. Biomechanics and Medicine in Swimming: Proceedings of the Fourth International Symposium of Biomechanics in Swimming and the Fifth International Congress on Swimming Medicine; 1982: Amsterdam: Human Kinetics Publishers, Inc.; 1983; pp. 123-35.

[3] Ikai M, Ishii K, Miyashita M. An electromyograhpic study of swimming. Res J Phys Educ 1964; 7(4): 47-54.

[4] Clarys JP, Rouard AH. The swimming muscle: history, methodology, and applications of electromyograhpy in swimming. In: Seifert L, Chollet D, Mujika I, Eds. World Book of Swimming: From Science to Performance. New York: Nova Science Publishers Inc. 2011; 43-68.

[5] Caty V, Rouard AH, Hintzy F, Aujouannet YA, Molinari F, Knafitz M. Time-frequency parameters of wrist muscles EMG after an exhaustive freestyle test. In: Vilas-Boas JP, Alves F, Marques A,
Eds. Biomechanics and Medicine in Swimming X: Proceedings of the Xth International Symposium on Biomechanics and Medicine in Swimming; 2006: Porto, Portugal: Portuguese Journal of Sport Sciences 2006; pp. 28-30.

[6] Lewillie L. Telemetrical analysis of the electromyogram. In: Wartenweiler J, Jokl E, Hebbelinck M, Eds. Biomechanics I; 1967: Zurich, Switzerland: S. Karger 1968; pp. 147-9.

[7] Okamoto T, Wolf SL. Underwater recording of electromyographic activity using fine-wire electrodes. In: Terauds J, Bedingfield EW, Eds. Swimming III, Proceedings of the Third international symposium of biomechanics in swimming; 1978: University of Alberta, Edmonton, Canada: University Park Press 1979; pp. 1606.

[8] Rainoldi A, Cescon C, Bottin A, Casale R, Caruso I. Surface EMG alterations induced by underwater recording. J Electromyogr Kinesiol 2004; 14(3): 325-31.

[9] Veneziano WH, da Rocha AF, Goncalves CA, et al. Confounding factors in water EMG recordings: an approach to a definitive standard. Med Biol Eng Comput 2006; 44(4): 348-51.

[10] Abbiss CR, Peiffer JJ, Netto KJ, Laursen PB. Reliability of surface EMG measurements of the quadriceps during maximal isometric contractions following water immersion. J Musculoskelet Res 2006; 10(4): 197-204.

[11] Nuber GW, Jobe FW, Perry J, Moynes DR, Antonelli D. Fine wire electromyography analysis of muscles of the shoulder during swimming. Am J Sports Med 1986; 14(1): 7-11.

[12] Delsys. Technical note 103: EMG signal analysis 2013 [cited 2013 31/01/2013]. Available from: www.delsys.com

[13] Masumoto K, Mercer JA. Biomechanics of human locomotion in water: an electromyographic analysis. Exerc Sport Sci Rev 2008; 36(3): 160-9.

[14] Poyhonen T, Keskinen KL, Hautala A, Savolainen J, Malkia E. Human isometric force production and electromyogram activity of knee extensor muscles in water and on dry land. Eur J Appl Physiol Occup Physiol 1999; 80(1): 52-6.

[15] Hermens HJ, Freriks B. European Recommendations for Surface ElectroMyoGraphy, results of the SENIAM project. Enschede: Roessingh Research and Development 1999.

[16] Konrad P. The ABC of EMG: A practical introduction to kinesiological electromyography. Scottsdale, AZ, USA: Noraxon USA Inc. 2006.

[17] Massó XA, Colado JC. Muscular activity of the posterior deltoid during swimming vs. resistance exercises on water and dry land. Int J Aquatic Res Educ 2010; 4: 61-9.

[18] Stirn I, Jarm T, Kapus V, Strojnik V. Evaluation of muscle fatigue during 100-m front crawl. Eur J Appl Physiol 2011; 111: 101-13. 